\title{
THE POTENTIAL IMPORTANCE OF SYNANTHROPIC VEGETATION TO BUMBLEBEES IN URBAN ECOSYSTEMS ON THE EXAMPLE OF LUBLIN
}

\author{
Magdalena Lubiarz', Ewa Trzaskowska² \\ The John Paul II Catholic University of Lublin \\ ${ }^{1}$ Department of Environmental Protection and Landscape Preservation \\ ${ }^{2}$ Department for Natural Fundations of landscape Architecture, Konstantynów 1H, 20-708 Lublin, Poland \\ e-mail: ${ }^{1}$ lubiarz@kul.pl, ${ }^{2}$ etrzaskowska@kul.pl
}

Received: 10.12.2013

\begin{abstract}
This study was an attempt to show synanthropic phytocoenoses of the city of Lublin which are potentially valuable to pollinating insects such as bumblebees. B. terrestris and $B$. lapidarius as well as $B$. lucorum, $B$. hypnorum and B. pascuorum were found to occur in Lublin. Artemisio-Tanacetetum, Bunietum orientalis, and Berteroëtum incanae were shown to be most valuable to bumblebees due to a high number of polleniferous and nectariferous species in these communities. The present study also allowed us to determine that the size of communities and their occurrence in a mosaic with parks and green spaces had an effect on the distribution of bumblebees.
\end{abstract}

Key words: synanthropic communities, bumblebees, urban ecosystems, urban vegetation

\section{Running head} bumblebees

Importance of urban synanthropic vegetation to

\section{INTRODUCTION}

Bumblebees belong to the family Apidae, tribe Bombini. In Europe 62 species are encountered [1], whereas 31 species belonging to the genus Bombus Latreille 1802 have been recorded in Poland [2]. Currently, most bumblebee species are fully protected in our country and only the buff-tailed bumblebee and red tailed bumblebee are partially protected under the relevant Regulation of the Minister of Environment [3]. Various authors stress the decrease in species richness and in the number of pollinating insects, including bumblebees [4-9].

Bumblebees occur in different environments, including urbanized environment [10-18]. Some forms of urban green spaces can be important resources for pollinators $[14,19,20]$. Even roadside vegetation can play a major role in the development of pollinating insects [21]. Many insects, including pollinators, use synanthropic plants as a source of food [5,7,22-25]. The importance of green areas as local fauna habitats is also increasing [17]. Even a small space overgrown with synanthropic vegetation is sometimes sufficient and can become a refuge for insects [26-28]. As reported by B a n a s z a k [29], a large dominance of social bee species is observed in cities. This author supposes that the social life of bees can be instrumental in overcoming the barriers of urbanization, similarly as polylectism, that is, an adaptation to forage on flowers of many unrelated plant species $[5,29,30]$. Bumblebees are both social and polylectic bees and hence they have traits that facilitate their survival in the anthropogenic environment. Nevertheless, A hrn é et al. [31] found in Stockholm a decline in the numbers of bumblebee species along a gradient of increasing urbanization, even in the case of the presence of a suitable habitat for foraging. In turn, $\mathrm{Paw} \mathrm{li} \mathrm{k} \mathrm{ow} \mathrm{s} \mathrm{k} \mathrm{i} \mathrm{and} \mathrm{O} l$ ę d z $\mathrm{k}$ a [32] showed in Torun that the mosaic nature of habitats and the proportion of developed land had the greatest effect on the occurrence of bumblebees in this city. Bumblebees prefer habitats with a large proportion of shrubs and in which developed land does not exceed $40 \%$ of the area [32]. Bumblebees build their nests mostly in the soil, but also on its surface between stones and plants, or in leaf litter. Most nests are observed on the edges of forests and tree stands as well as under shrubs, in field margins or in clover crops [5,7]. Both foraging flight ranges and energy requirements of large and small bees differ $[33,34]$. 
As a result of that, each of these groups responds differently to environmental changes at the landscape level. Their sensitivity to the specific characteristics of a particular habitat also varies [35,36]. Large social bees, including bumblebees, are capable of foraging within a radius of more than $1 \mathrm{~km}$ from their nests, whereas smaller solitary bees are generally thought to be able to fly to a distance of about $250 \mathrm{~m}$ from their nests $[20,34,37,38]$. However, E r e $\mathrm{m}$ e e v a and $\mathrm{S} \mathrm{u}$ $\mathrm{shchev}$ [25] report that bumblebees look for food within a radius of $2 \mathrm{~km}$ from their nests. In turn, $\mathrm{W}$ o $1 \mathrm{f}$ and M o r i t z [39] showed that Bombus terrestris workers foraged within an average distance of $267 \mathrm{~m}$ from their nests, $800 \mathrm{~m}$ at the maximum. Green $\mathrm{le}$ af et al. [34] give a method for measuring foraging distance of Apidae depending on body size, but the presented results mainly relate to non-social bees. However, these authors did not determine whether trophic specialization (including polylectism) affected bee foraging distance [34].

In the Lublin region, there have been earlier studies on the importance of plants to pollinating insects. M a s i e r ow ska [40] studied in Lublin ornamental plants of the family Saxifragaceae as a source of food for pollinating insects. Wrzesień and Den is ow $[26,41,42]$ investigated the proportion of bee plants in xerothermic grasslands of the Lublin Upland and in phytocoenoses near railway tracks of the Lublin Upland as well as in the Roztocze and Polesie regions. De n i s ow and Wrzesién [43] investigated midfield tree stands, fallow lands and field margins in the area of Lublin, with special attention to nectariferous and polleniferous plants.

The aim of the present study was to show the role of synanthropic communities to bumblebees found in urbanized areas on the example of Lublin. Biodiversity conservation provides for measures at all levels and in all kinds of environments. Therefore, the present authors decided to investigate the potential importance of spontaneous vegetation of urban areas in the maintenance of biological diversity of this important group of pollinating insects, notably bumblebees.

\section{MATERIALS AND METHODS}

Phytosociological surveys covering the Lublin city area formed the basis for this study designed to determine the importance of synanthropic vegetation to bumblebees. 386 relevés were made over the period 2005-2010. Field investigations were carried out using relevés which were analysed following the Braun-Blanquet method. During the investigations, bumblebee visitation and foraging were observed. In May and June 2012, observations were carried out in individual synanthropic communities to record in which of them bumblebees appeared and in which they did not occur.
These were only qualitative investigations, not quantitative ones. Particular individuals were not counted and only the presence of bumblebees in a particular type of environment was recorded. This allowed us to initially determine whether synanthropic communities of Lublin were used by bumblebees at all. Bumblebees were identified using the relevant keys $[7,44]$.

Subsequently, the collected material was analysed in terms of the potential usefulness of the identified communities and plants composing these communities to bumblebees. The distribution and size of synanthropic vegetation patches in Lublin were analysed. Synthetic vegetation tables were made and they showed the following indices: cover-abundance, sociability and constancy. The phenology of synanthropic plant species occurring in synanthropic communities of Lublin was determined. The data relating to plants useful to pollinating insects (nectariferous and polleniferous species) followed $[26,45]$. The obtained phytosociological data were compared with the phenology of the two most frequently observed bumblebee species, Bombus terrestris and Bombus lapidarius. In this way, the study demonstrated the potential usefulness of synanthropic plant communities in Lublin as a food resource for bumblebees. This paper follows phytosociological nomenclature of Matuszkiewicz [46] and it is additionally based on the studies of Fijałkow ski [47] and J an ecki [48]. The names of bumblebees follow B a n s z ak [2].

\section{RESULTS}

The present study conducted in Lublin found the occurrence of 35 synanthropic communities: Vicietum tetrospermae, Galinsogo-Setarietum, Echinochloo-Setarietum, Panico-Eragrostietum, Chenopodietum stricti, Sisymbrietum loeselii, Urtico-Malvetum, Erigeronto-Lactucetum, Hordeetum murini, a community with Lepidum ruderale, Onopordetum acanthii, Echio-Melilotetum, Berteroëtum incanae, Artemisio-Tanacetetum, Bunietum orientalis, Leonuro-Ballotetum, Leonuro-Arctietum, Ivetum xantifoliae, Tussilaginetum, a community with Cannabis ruderalis, a community with $\mathrm{He}$ liantus tuberosus, Urtico-Aegopodietum, a community with Impatiens parviflora, Chelidonio-Robinietum, $\mathrm{Ca}$ lystegio-Eupatorietum, Urtico-Calystegietum, a community with Impatiens glandulifera, a community with Lycium barbarum, a community with Reynoutria japonica, Sambucetum nigrae, Cardario-Agropyretum, Lolio-Polygonetum, Lolio-Potentilletum, Prunello-Plantaginetum, and Bryo-Saginetum procumbentis (Table 1).

The presence of bumblebees was recorded in most synanthropic communities of Lublin. These insects were not observed only in the community with Lepidum ruderale, Chenopodietum stricti, Panico-Eragrostietum, and Bryo-Saginetum. The following 
bumblebee species were found to occur within the study area: Bombus terrestris (Linnaeus 1758), Bombus lapidarius (Linnaeus 1758), Bombus lucorum (Linnaeus 1761), Bombus hypnorum (Linnaeus 1758), and Bombus pascuorum (Scopoli 1763). The other bumblebees that appeared within the study area were not identified to the level of species. Two species: $B$. terrestris and B. lapidaries, were most frequently observed. Given that bumblebees foraging in synanthropic communities were observed in Lublin, it should therefore be assumed that a part of them can also nest within the city area, including in synanthropic communities where shrubs or clusters of plants such as Tanacetum vulgare are observed.

The pattern of distribution and variation of synanthropic communities in Lublin can be related to the occurrence of bumblebees. These insects are associated with areas in which they can build nests and at the same time find food $[49,50]$. As shown by the research, the spatial pattern of communities is determined by anthropogenic factors manifested in the zonation from the centre of the city to its outskirts and this is associated with the historically determined distribution of anthropopressure. Different zones can be distinguished, which is also confirmed by the study on vegetation conducted by $\mathrm{R} \mathrm{y} \mathrm{s} \mathrm{i} \mathrm{a} \mathrm{k} \mathrm{[51].}$

The smallest spaces are occupied by synanthropic plants and communities in the city centre where at the same time green areas are also the smallest. They are limited to degraded lawns and slopes. There are communities with low specialization (Lolio-Polygonetum, Bryo-Saginetum procumbentis, a community with Lycium barbarum), communities in impoverished form (a smaller number of species recorded in these communities compared to those found on the city outskirts), and communities that often occur only temporarily $(\mathrm{Pa}$ nico-Eragrostietum), which is associated with their destruction during tending treatments. Single bumblebee individuals were observed there, in particular on the slope of the Old Town near allotment gardens located on the Bystrzyca River.

The variation in plants and communities increases with an increasing distance from the city centre and already in the Śródmieście district synanthropic communities occupy larger area; apart from the above-mentioned ones, communities associated with parks, green spaces, and flower borders appear (Galinsogo-Setarietum, Urtico-Malvetum, Hordeetum murini, Urtico-Aegopodietum, a community with Impatiens parviflora, Chelidonio-Robinietum). Here, there are also no connections between individual spaces overgrown with plants. As indicated above, single bumblebee individuals were recorded in these communities.

On the other hand, in housing estates, near roads and on bodies of water, synanthropic plants and communities are an admixture and they form a mosaic together with structured vegetation and natural communities. In terms of area, synanthropic communities are a dominant component of green spaces (extensive lawns). 23 synanthropic communities were identified in this part of the city. Among them, the following were frequently encountered: Bunietum orientalis, Cardario-Agropyretum, Chelidonio-Robinietum, and Urtico-Aegopodietum. They usually develop near cultural landscape elements: consciously structured plant cover or artificial abiotic structures. This variation in communities and the occurrence of a mosaic had a distinct effect on the presence of bumblebees, in particular at places where wastelands and extensive lawns were adjacent to each other.

Synanthropic communities are a major element of the landscape in undeveloped areas and wastelands. Communities found in such places are characterized by high species variation and they occupy an area from $25 \mathrm{~m}$, to several hectares [52]. For many years, the development of ruderal communities has been observed near railway tracks and in post-industrial areas that have not yet been designated for any specific use as well as in managed green spaces such as lawns and parks. Such wastelands overgrown primarily with Artemisio-Tanacetetum can be found in almost all the gullies of Lublin, but also in fallow lands in a mosaic with cultivated fields on the city outskirts. Ruderal communities also occupy the largest areas in Lublin, though the spread of these communities is limited by changes in land use, progressive urbanization, the development of wastelands, and the aestheticization of space. Bumblebees were most often observed in such places and these were always numerous individuals.

In Lublin the variation of synanthropic communities and the area occupied by them affected the occurrence of bumblebees. This is also shown in the research of $\mathrm{Paw}$ li kow s ki and $\mathrm{Oleqdzka} \mathrm{[32]} \mathrm{as}$ well as in the study of Ere mee va and S u s h che v [25]. Bumblebees prefer urban areas with mosaic vegetation, but with a predominance of shrubby vegetation, and they nest within a distance of $2 \mathrm{~km}$ from foraging sites. They choose areas with woody or grassland vegetation much less frequently [25,32]. To build nests, bumblebee species occurring in Poland need areas covered by shrubs or with dense canopies of large perennials. A part of species nest on the edges of tree stands [5]. Only some species, such as Bombus hypnorum, are able to locate their nests in gaps between stones or in attics of residential and industrial buildings [25].

Spontaneous synanthropic communities develop to different degrees and their floristic composition significantly differ from the structure of communities known from typical ruderal sites (wastelands, landfills, places near human dwellings and fences, etc.) in, among others, the proportion of meadow species and in the absence of characteristic synanthropic species. 
The analysis of the status of synanthropic communities in Lublin shows that urbanization results not only in decreased variation in plant communities, but it also causes a decline in the number of species. Resistant species, in particular cosmopolitan ones (Eragrostis sp., Plantago major, Polygonum aviculare), which form small clusters and are of little importance to bumblebees, are predominant in the city centre. The further from the centre, the variation in anthropopressure forms increases the richness of plant communities and the number of species in the external zone of Lublin also increases. In terms of species variation, the least numerous were associations of the class Molinio-Arrhenatheretea Bryo-Saginetum in which 17 species were identified (on average 4 species per relevé) and of the class Stelarietea mediae - a community with Lepidium ruderale, in which 11 species were identified (on average 7); they were found in habitats most transformed by anthropopressure in the city centre. The following communities of the class Artemisietea vulgaris were richest in species: Artemisio-Tanacetetum in which 170 species were found (on average 17) and Bunietum orientalis with 110 species (on average 18), occurring in the gullies, wastelands, and extensive lawns. These are also communities richest in nectar and pollen producing species (Table 1). The largest concentrations of bee plants were found in the following communities: Atremisio-Tanacetetum (60 species), Bunietum orientalis (46 species), and Berteroëtum incanae (41 species). Half less polleniferous and nectariferous species were found in the following communities: Sisymbrietum loeselii (27 species), Urtico-Calystegietum (21 species), Urtico-Malvetum (20 species), Echio-Melilotetum (20 species), and Leonuro-Ballotetum (20 species). The percentage of bee forage species found in individual communities is from 14 to $100 \%$, whereas in nine communities they accounted for more than $50 \%$ of the species composition. However, this does not coincide with the variation in the communities in terms of their species richness. In spite of great species variation in synanthropic communities, only a part of plant species important to bumblebees occur frequently or with high cover-abundance and sociability in synanthropic communities of Lublin, which was determined taking into account the constancy classes (Table 2). The following can be mentioned among them: Achillea millefolium L. (17 associations), Aegopodium podagraria L. (8 associations), Artemisia vulgaris L. (22 associations), Ballota nigra L. (17 associations), Chelidonium majus L. (7 associations), Chenopodium album L. (12 associations), Galium aparine L. (13 associations), Lamium album L. (11 associations), Medicago lupulina L. (9 associations), Melandrium album (Mill.) Garcke (11 associations), Sambucus nigra L. (9 associations), Solidago gigantea Aiton (15 associations), Tanacetum vulgare
L. (12 associations), Taraxacum officinale F.H. Wigg. (27 associations), and Trifolium pratense L. (10 associations). At the same time, it was observed that there were communities, e.g. Erigeronto-Lactucetum, Panico-Eragrostietum, Ivetum xantifoliae, and a community with Impatiens glandulifera, in which bee plants were most frequently characterized by low cover. Besides, the species found in them do not provide for the needs of insects throughout the whole season.

Another aspect that was taken into consideration was the flowering duration of bee plants in particular communities and clusters formed by them. The analysis of the presence of bee forage plants in particular communities and their flowering periods shows that the above-mentioned plants formed dense clusters and their flowering occurred in different periods of the growing season in the following communities: Atremisio-Tanacetetum, Bunietum orientalis, and Berteroëtum incanae (Tables 2 and 3).

The results of the present study compared with the phenology of the two most numerous bumblebee species confirm the potential usefulness of synanthropic plant communities to these insects. In particular, the flowering periods of synanthropic bee plants clearly coincide with the periods of dynamic bumblebee development (Tables 2-4). Plants of synanthropic communities such as Tussilago farfara or Trifolium repens flower already from April or May. In turn, Ballota nigra and Medicago sp. flower from June even until the end of September (Table 2). The strongest bumblebee colony development usually occurs between June and August (Table 4), which coincides with the flowering of many plants valuable to these insects or of one species but which is a dominant element in the community. It is perfectly reflected in Table 3 which shows the flowering of bee plants in synanthropic communities of Lublin. The dominant (full) flowering occurs in particular in June, July and August, thus in the months important in bumblebee colony development. Taking into account both the number of bee forage species in synanthropic communities of Lublin and the full flowering period of these plants, it can be stated that the communities Artemisio-Tanacetetum and Bunietum orientalis have the greatest importance as a source of food for bumblebees, even more so that these communities occupy larger areas and occur in a mosaic with other communities. We should not however ignore the importance of other communities that can be a supplementary source of food, since bumblebees are polylectic insects. Given the trophic adaptations of bumblebees, it can be concluded that synanthropic communities are potentially very valuable to them. In the spring and early summer, these insects readily visit flowers of trees such as Robinia pseudoacacia or Tilia cordata as a source of food, but the period of late summer or 
early autumn is not abundant in flowering trees and album have a huge role in providing food to bumbletherefore species such as Galium aparine or Lamium bees after these trees finish flowering.

Table 1

Species variation in synanthropic communities found in Lublin, together with the number and percentage of bee plant species

\begin{tabular}{|c|c|c|c|c|}
\hline No. & Name of association & $\begin{array}{l}\text { Number of plant species } \\
\text { in particular synanthropic } \\
\text { communities }\end{array}$ & $\begin{array}{l}\text { Number of bee } \\
\text { plant species }\end{array}$ & $\begin{array}{l}\text { Percentage of bee } \\
\text { plant species }\end{array}$ \\
\hline 1. & Vicietum tetrospermae & 64 & 19 & 29.7 \\
\hline 2. & Echinochloo-Setarietum & 39 & 18 & 46.1 \\
\hline 3. & Galinsogo-Setarietum & 30 & 14 & 46.7 \\
\hline 4. & Panico-Eragrostietum & 25 & 13 & 52.0 \\
\hline 5. & Chenopodietum stricti & 31 & 15 & 48.4 \\
\hline 6. & Sisymbrietum loeselii & 49 & 27 & 55.1 \\
\hline 7. & Urtico-Malvetum & 59 & 20 & 33.9 \\
\hline 8. & Erigeronto-Lactucetum & 8 & 8 & 100 \\
\hline 9. & Hordeetum murini & 42 & 14 & 33.3 \\
\hline 10. & community with Lepidium ruderale & 10 & 6 & 60 \\
\hline 11. & Onopordetum acanthii & 13 & 8 & 61.5 \\
\hline 12. & Echio-Melilotetum & 52 & 20 & 38.5 \\
\hline 13. & Berteroëtum incanae & 73 & 41 & 56.2 \\
\hline 14. & Artemisio-Tanacetetum & 170 & 60 & 35.3 \\
\hline 15. & Bunietum orientalis & 110 & 46 & 41.8 \\
\hline 16. & Leonuro-Ballotetum & 48 & 20 & 41.7 \\
\hline 17. & Leonuro-Arctietum & 24 & 14 & 58.3 \\
\hline 18. & Ivetum xantifolia & 25 & 13 & 52.0 \\
\hline 19. & Tussilaginetum & 33 & 8 & 24.2 \\
\hline 20. & community with Cannabis ruderale & 20 & 8 & 40.0 \\
\hline 21. & community with Heliantus tuberosus & 35 & 5 & 14.3 \\
\hline 22. & Urtico-Aegopodietum & 49 & 19 & 38.8 \\
\hline 23. & community with Impatiens parviflora & 41 & 9 & 21.9 \\
\hline 24. & Chelidonio-Robinietum & 54 & 15 & 27.8 \\
\hline 25. & Calystegio-Eupatorietum & 31 & 14 & 45.2 \\
\hline 26. & Urtico-Calystegietum & 47 & 21 & 44.7 \\
\hline 27. & community with Impatiens glandulifera & 7 & 7 & 100 \\
\hline 28. & community with Lycium barbarum & 55 & 16 & 29.1 \\
\hline 29. & community with Reynoutria japonica & 28 & 8 & 28.6 \\
\hline 30. & Sambucetum nigrae & 42 & 14 & 33.3 \\
\hline 31. & Cardario-Agropyretum & 68 & 17 & 25.0 \\
\hline 32. & Lolio-Polygonetum & 86 & 12 & 14.0 \\
\hline 33. & Lolio-Potentilletum & 34 & 9 & 26.5 \\
\hline 34. & Prunello-Plantaginetum & 46 & 15 & 32.6 \\
\hline 35. & Bryo-Saginetum & 17 & 6 & 35.3 \\
\hline
\end{tabular}


Table 2.

List and characteristics of plant species most frequently found in synanthropic communities in Lublin

\begin{tabular}{|c|c|c|c|c|}
\hline No. & Plant species & $\begin{array}{l}\text { Usefulness } \\
\text { of plants to } \\
\text { bumblebees }\end{array}$ & $\begin{array}{l}\text { Average } \\
\text { flowering time }\end{array}$ & $\begin{array}{l}\text { Occurrence of the species } \\
\text { in synanthropic communities }{ }^{2} \\
\left.\text { (Cover-abundance }^{3} \text {; Sociability }{ }^{4}\right)\end{array}$ \\
\hline 1. & Acer negundo L. & $\mathrm{N}, \mathrm{P}$ & $05.04-20.05$ & $\begin{array}{l}\mathbf{5}(1,1), \mathbf{1 1}(1,1), \mathbf{1 4}(1,1), \mathbf{1 5}(1,1), \mathbf{1 6}(1,1), \mathbf{1 7}(1,1), \mathbf{2 4}(1,3), \\
\mathbf{2 6}(1,1), \mathbf{2 8}(1,1)\end{array}$ \\
\hline 2. & Achillea millefolium $\mathrm{L}$. & $\mathrm{N}, \mathrm{P}$ & $20.05-30.09$ & $\begin{array}{l}\mathbf{1}(1,2), \mathbf{3}(1,1), \mathbf{4}(1,4), \mathbf{7}(2,2), \mathbf{8}(1,1), \mathbf{9}(1,2), \mathbf{1 2}(1,4), \\
\mathbf{1 3}(1,3), \mathbf{1 4}(1,3), \mathbf{1 5}(1,3), \mathbf{2 8}(1,2), \mathbf{3 0}(1,1), \mathbf{3 1}(1,1), \mathbf{3 2}(1,2), \\
\mathbf{3 3}(1,1), \mathbf{3 4}(1,3), \mathbf{3 5}(1,1)\end{array}$ \\
\hline 3. & Aegopodium podagraria $\mathrm{L}$. & $\mathrm{N}, \mathrm{P}$ & $20.05-10.07$ & $\begin{array}{l}\mathbf{1 5}(1,1), \mathbf{2 0}(3,1), \mathbf{2 2}(3,5), \mathbf{2 3}(3,3), \mathbf{2 4}(2,3), \mathbf{2 6}(2,1), \mathbf{2 9}(1,2) \text {, } \\
\mathbf{3 0}(2,2)\end{array}$ \\
\hline 4. & Agrimonia eupatoria $\mathrm{L}$. & $\mathrm{N}, \mathrm{P}$ & $10.06-15.08$ & $\mathbf{1 4}(1,1)$ \\
\hline 5. & Anchusa officinalis L. & $\mathrm{N}, \mathrm{P}$ & $15.05-30.09$ & $\mathbf{1 5}(1,1)$ \\
\hline 6. & Anthemis arvensis L. & $\mathrm{N}, \mathrm{P}$ & $15.06-20.07$ & $\mathbf{1}(1,2), \mathbf{2}(1,2), \mathbf{3}(1,3), \mathbf{6}(2,2)$ \\
\hline 7. & Arctium lappa $\mathrm{L}$. & $\mathrm{N}, \mathrm{P}$ & $10.07-20.08$ & $\begin{array}{l}\mathbf{9}(1,2), \mathbf{1 4}(1,1), \mathbf{1 5}(1,4), \mathbf{1 6}(2,4), \mathbf{1 7}(1,1), \mathbf{2 6}(1,2), \mathbf{2 8}(1,1), \\
\mathbf{3 1}(1,1)\end{array}$ \\
\hline 8. & Arctium minus (Hill) Bernh. & $\mathrm{N}, \mathrm{P}$ & $05.07-20.08$ & $\mathbf{1 8}(1,2), \mathbf{2 2}(1,2), \mathbf{2 3}(1,1), \mathbf{2 4}(1,1), \mathbf{3 0}(1,1)$ \\
\hline 9. & Arctium tomentosum Mill. & $\mathrm{N}, \mathrm{P}$ & $01.07-01.09$ & $\mathbf{5}(1,2), \mathbf{7}(1,1), \mathbf{1 4}(1,1), \mathbf{1 5}(1,1), \mathbf{2 5}(1,1)$ \\
\hline 10 & Artemisia vulgaris L. & $\mathrm{P}$ & $15.07-20.10$ & $\begin{array}{l}\mathbf{1}(1,2), \mathbf{2}(1,2), \mathbf{3}(1,4), \mathbf{4}(1,1), \mathbf{5}(1,2), \mathbf{6}(1,1), \mathbf{7}(1,1), \mathbf{1 2}(1,4) \\
\mathbf{1 3}(1,2), \mathbf{1 4}(1,5), \mathbf{1 5}(1,2), \mathbf{1 6}(1,2), \mathbf{1 7}(1,2), \mathbf{1 8}(1,2), \mathbf{2 2}(1,4) \\
\mathbf{2 4}(1,1), \mathbf{2 5}(1,2), \mathbf{2 6}(1,1), \mathbf{2 7}(1,1), \mathbf{2 8}(1,3), \mathbf{3 1}(1,3), \mathbf{3 2}(1,1)\end{array}$ \\
\hline 11. & Aster novi-belgii $\mathrm{L}$. & $\mathrm{N}, \mathrm{P}$ & $10.08-20.09$ & $\mathbf{2 6}(1,1)$ \\
\hline 12. & Ballota nigra $\mathrm{L}$. & $\mathrm{N}, \mathrm{P}$ & $01.07-10.09$ & $\begin{array}{l}\mathbf{6}(1,1), \mathbf{7}(1,2), \mathbf{9}(1,1), \mathbf{1 3}(2,1), \mathbf{1 5}(1,1), \mathbf{1 6}(2,4), \mathbf{1 7}(2,4), \\
\mathbf{1 8}(1,2), \mathbf{1 9}(1,1), \mathbf{2 0}(1,2), \mathbf{2 1}(1,1), \mathbf{2 2}(1,1), \mathbf{2 3}(1,2), \mathbf{2 4}(1,4), \\
\mathbf{2 6}(1,1), \mathbf{2 7}(1,4), \mathbf{3 0}(1,1)\end{array}$ \\
\hline 13. & Bellis perennis L. & $\mathrm{N}, \mathrm{P}$ & $20.04-15.07$ & $\mathbf{3 4}(1,1)$ \\
\hline 14. & Berteroa incana (L.) DC. & $\mathrm{N}, \mathrm{P}$ & $10.05-30.09$ & $\mathbf{4}(1,1), \mathbf{1 2}(1,1), \mathbf{1 3}(1,5), \mathbf{1 4}(1,1), \mathbf{1 5}(1,1), \mathbf{2 5}(1,1)$ \\
\hline 15. & Bunias orientalis L. & $\mathrm{N}, \mathrm{P}$ & $05.05-10.06$ & $\mathbf{6}(1,1), \mathbf{1 5}(4,5)$ \\
\hline 16. & Calystegia sepium (L.) R.Br. & $\mathrm{N}, \mathrm{P}$ & $10.06-10.09$ & $\mathbf{1 8}(1,1), \mathbf{2 5}(4,5), \mathbf{2 6}(3,5), \mathbf{2 7}(3,1)$, \\
\hline 17. & Cardaria draba (L.) Desv. & $\mathrm{N}, \mathrm{P}$ & $05.05-01.06$ & $\mathbf{1 8}(1,1), \mathbf{3 1}(3,5)$ \\
\hline 18. & Carduus crispus L. & $\mathrm{N}, \mathrm{P}$ & $25.06-01.09$ & $\mathbf{1 5}(2,1)$ \\
\hline 19. & Centaurea cyanus L. & $\mathrm{N}, \mathrm{P}$ & $10.06-01.08$ & $\mathbf{2}(2,3), \mathbf{1 3}(1,1)$ \\
\hline 20. & Centaurea scabiosa $\mathrm{L}$. & $\mathrm{N}, \mathrm{P}$ & $20.06-10.09$ & $\mathbf{1 3}(2,1)$ \\
\hline 21. & $\begin{array}{l}\text { Chamomilla suaveolens } \\
\text { (Pursh) Rydb. }\end{array}$ & $\mathrm{N}, \mathrm{P}$ & $10.06-20.07$ & $\begin{array}{l}\mathbf{4}(1,1), \mathbf{6}(1,1), \mathbf{7}(1,1), \mathbf{9}(1,2), \mathbf{1 0}(1,1), \mathbf{1 6}(1,2), \mathbf{3 2}(1,4), \\
\mathbf{3 3}(1,2), \mathbf{3 4}(1,1), \mathbf{3 5}(1,2)\end{array}$ \\
\hline 22. & Chelidonium majus L. & $\mathrm{P}$ & $05.05-10.10$ & $\mathbf{1 3}(1,1), \mathbf{1 7}(1,1), \mathbf{2 2}(2,2), \mathbf{2 3}(1,1), \mathbf{2 4}(3,5), \mathbf{2 8}(1,2), \mathbf{3 0}(2,3)$ \\
\hline 23. & Chenopodium album $\mathrm{L}$. & $\mathrm{P}$ & $20.06-20.09$ & $\begin{array}{l}\mathbf{3}(1,4), \mathbf{4}(1,1), \mathbf{5}(2,5), \mathbf{6}(1,3), \mathbf{7}(1,1), \mathbf{8}(1,1), \mathbf{9}(1,1), \mathbf{1 6}(1,2), \\
\mathbf{1 8}(1,1), \mathbf{2 0}(1,2), \mathbf{2 9}(1,1), \mathbf{3 3}(1,1)\end{array}$ \\
\hline 24. & Cichorium intybus L. & $\mathrm{N}, \mathrm{P}$ & $10.06-01.09$ & $\mathbf{1 2}(1,1), \mathbf{1 4}(1,1), \mathbf{1 5}(1,2), \mathbf{3 1}(1,2)$ \\
\hline 25. & Cirsium arvense (L.) Scop. & $\mathrm{N}, \mathrm{P}$ & $30.06-20.08$ & $\begin{array}{l}\mathbf{2}(1,2), \mathbf{3}(1,1), \mathbf{5}(1,2), \mathbf{8}(1,2), \mathbf{1 2}(1,4), \mathbf{1 4}(1,4), \mathbf{1 5}(1,3), \\
\mathbf{1 6}(1,1), \mathbf{1 7}(1,2), \mathbf{1 8}(1,1), \mathbf{1 9}(1,2), \mathbf{2 2}(1,2), \mathbf{2 5}(1,1), \mathbf{2 6}(1,3), \\
\mathbf{2 7}(1,1), \mathbf{3 1}(1,2)\end{array}$ \\
\hline 26. & Consolida regalis Gray & $\mathrm{N}, \mathrm{P}$ & $10.06-20.07$ & $\mathbf{1}(1,1), \mathbf{2}(1,1), \mathbf{3}(1,1)$ \\
\hline 27. & Convolvulus arvensis L. & $\mathrm{N}, \mathrm{P}$ & $10.06-10.09$ & $\begin{array}{l}\mathbf{1}(1,4), \mathbf{2}(1,2), \mathbf{3}(1,4), \mathbf{5}(1,2), \mathbf{7}(2,2), \mathbf{9}(1,3), \mathbf{1 0}(1,1), \mathbf{1 1}(2,1), \\
\mathbf{1 2}(1,1), \mathbf{1 3}(1,1), \mathbf{1 4}(1,2), \mathbf{1 5}(1,2), \mathbf{1 6}(1,1), \mathbf{2 2}(1,1), \mathbf{3 1}(1,4), \\
\mathbf{3 2}(1,1)\end{array}$ \\
\hline 28. & $\begin{array}{l}\text { Conyza canadensis }(\mathrm{L} .) \\
\text { Conqist }\end{array}$ & $\mathrm{P}$ & $05.06-10.10$ & $\begin{array}{l}\mathbf{1}(1,1), \mathbf{2}(1,1), \mathbf{3}(1,1), \mathbf{4}(1,2), \mathbf{5}(1,2), \mathbf{6}(1,1), \mathbf{7}(1,4), \mathbf{8}(1,4), \\
\mathbf{9}(1,2), \mathbf{1 0}(1,2), \mathbf{1 4}(1,1), \mathbf{1 5}(1,1), \mathbf{1 6}(1,2), \mathbf{2 6}(1,1), \mathbf{2 8}(1,2), \\
\mathbf{3 1}(1,2), \mathbf{3 2}(1,2), \mathbf{3 5}(1,2)\end{array}$ \\
\hline 29. & Coronilla varia $\mathrm{L}$. & $\mathrm{N}, \mathrm{P}$ & $10.06-10.08$ & $\mathbf{1 3}(2,1)$ \\
\hline 30. & Daucus carota $\mathrm{L}$. & $\mathrm{N}, \mathrm{P}$ & $20.06-15.09$ & $\mathbf{1}(1,2), \mathbf{5}(1,2), \mathbf{8}(1,2), \mathbf{1 2}(1,5), \mathbf{1 3}(1,2), \mathbf{1 4}(1,3)$ \\
\hline 31. & Echium vulgare L. & $\mathrm{N}, \mathrm{P}$ & $10.06-10.09$ & $\mathbf{1 2}(2,4), \mathbf{1 3}(1,2), \mathbf{1 5}(1,2)$ \\
\hline
\end{tabular}




\begin{tabular}{|c|c|c|c|c|}
\hline 32. & Epilobium angustifolium $\mathrm{L}$. & $\mathrm{N}$ & $10.06-15.09$ & $\mathbf{1 4}(1,1)$ \\
\hline 33. & Eupatorium cannabinum $\mathrm{L}$. & $\mathrm{N}, \mathrm{P}$ & $20.06-15.08$ & $\mathbf{2 5}(3,5)$ \\
\hline 34. & Euphorbia cyparissias L. & $\mathrm{N}, \mathrm{P}$ & $01.05-20.05$ & $\mathbf{1 3}(1,1), \mathbf{1 5}(1,1), \mathbf{1 8}(1,1)$ \\
\hline 35. & Euphorbia esula L. & $\mathrm{N}, \mathrm{P}$ & $20.05-20.07$ & $\mathbf{1 2}(1,1), \mathbf{1 4}(1,1)$ \\
\hline 36. & $\begin{array}{l}\text { Fallopia convolvulus (L.) } \\
\text { A. Love }\end{array}$ & $\mathrm{N}, \mathrm{P}$ & $20.05-20.06$ & $\mathbf{1}(1,1), \mathbf{3}(1,2), \mathbf{1 3}(1,1), \mathbf{2 5}(1,1), \mathbf{2 6}(1,2)$ \\
\hline 37. & Galeopsis tetrahit $\mathrm{L}$. & $\mathrm{N}$ & $15.06-10.10$ & $\mathbf{6}(1,1), \mathbf{1 3}(1,1)$ \\
\hline 38. & Galium aparine $\mathrm{L}$. & $\mathrm{N}, \mathrm{P}$ & $10.06-15.09$ & $\begin{array}{l}\mathbf{1}(1,1), \mathbf{2}(1,2), \mathbf{1 3}(1,1), \mathbf{1 4}(1,1), \mathbf{1 5}(1,1), \mathbf{1 6}(1,1), \mathbf{1 7}(1,3), \\
\mathbf{2 0}(2,2), \mathbf{2 2}(3,3), \mathbf{2 4}(2,2), \mathbf{2 5}(3,1), \mathbf{2 6}(1,2), \mathbf{2 8}(1,2)\end{array}$ \\
\hline 39. & Galium mollugo L. & $\mathrm{N}$ & $15.06-15.09$ & $\mathbf{1 3}(1,1), \mathbf{1 4}(1,1), \mathbf{1 5}(1,2), \mathbf{2 5}(1,2)$ \\
\hline 40. & Galium verum $\mathrm{L}$. & $\mathrm{N}, \mathrm{P}$ & $10.07-20.09$ & $\mathbf{1 4}(1,1)$ \\
\hline 41. & Geranium pratense $\mathrm{L}$. & $\mathrm{N}, \mathrm{P}$ & $10.06-10.08$ & $\mathbf{1 4}(1,1)$ \\
\hline 42. & Geum urbanum L. & $\mathrm{N}, \mathrm{P}$ & $01.06-20.06$ & $\mathbf{1 3}(1,1), \mathbf{2 2}(1,4), \mathbf{2 3}(1,3), \mathbf{2 9}(1,1), \mathbf{3 0}(1,1)$ \\
\hline 43. & Glechoma hederacea $\mathrm{L}$. & $\mathrm{N}, \mathrm{P}$ & $20.04-10.07$ & $\mathbf{7}(1,1), \mathbf{2 2}(1,2)$ \\
\hline 44. & Helianthus tuberosus L. & $\mathrm{N}, \mathrm{P}$ & $20.08-15.10$ & $\mathbf{5}(1,2), \mathbf{2 1}(3,5)$ \\
\hline 45 . & Heracleum sphondylium $\mathrm{L}$. & $\mathrm{N}, \mathrm{P}$ & $15.06-01.09$ & $\mathbf{1 4}(1,1), \mathbf{1 5}(1,1), \mathbf{2 5}(1,2), \mathbf{2 6}(1,2)$ \\
\hline 46. & Hypercium perforatum $\mathrm{L}$. & $\mathrm{P}$ & $05.06-30.07$ & $\mathbf{1 2}(1,1), \mathbf{1 4}(1,1), \mathbf{1 5}(1,1), \mathbf{3 4}(1,1)$ \\
\hline 47. & Impatiens glandulifera Royle & $\mathrm{N}, \mathrm{P}$ & $20.07-30.09$ & $27(2,5)$ \\
\hline 48. & Impatiens parviflora DC. & $\mathrm{N}, \mathrm{P}$ & $20.07-10.09$ & $\begin{array}{l}\mathbf{6}(1,1), \mathbf{1 3}(1,1), \mathbf{2 2}(1,2), \mathbf{2 3}(4,5), \mathbf{2 4}(3,3), \mathbf{2 5}(1,1), \mathbf{2 6}(1,3) \\
\mathbf{2 8}(1,2), \mathbf{3 0}(1,1)\end{array}$ \\
\hline 49. & Iva xantiifolia Nutt. & $\mathrm{P}$ & $20.08-20.10$ & $\mathbf{5}(1,1), \mathbf{1 8}(4,5)$ \\
\hline 50 . & Lamium album $\mathrm{L}$. & $\mathrm{N}, \mathrm{P}$ & $20.04-30.09$ & $\begin{array}{l}\mathbf{6}(1,1), \mathbf{7}(1,1), \mathbf{1 5}(1,1), \mathbf{1 6}(1,2), \mathbf{1 7}(1,1), \mathbf{2 2}(1,1), \mathbf{2 3}(1,1), \\
\mathbf{2 4}(1,1), \mathbf{2 5}(1,1), \mathbf{2 6}(1,1), \mathbf{3 0}(1,1)\end{array}$ \\
\hline 51. & Lamium purpureum $\mathrm{L}$. & $\mathrm{N}, \mathrm{P}$ & $15.04-01.09$ & $\mathbf{3}(1,2)$ \\
\hline 52. & Leonurus cardiaca $\mathrm{L}$. & $\mathrm{N}, \mathrm{P}$ & $20.06-15.08$ & $\begin{array}{l}\mathbf{1 5}(1,1), \mathbf{1 7}(2,4), \mathbf{2 2}(1,1), \mathbf{2 4}(1,2), \mathbf{2 5}(1,1), \mathbf{2 6}(1,2), \mathbf{2 8}(1,2) \text {, } \\
\mathbf{3 0}(1,1)\end{array}$ \\
\hline 53. & Linaria vulgaris Mill. & $\mathrm{N}, \mathrm{P}$ & $15.06-20.09$ & $\mathbf{1}(1,1), \mathbf{4}(1,1), \mathbf{6}(1,1)$ \\
\hline 54. & Lotus corniculatus L. & $\mathrm{P}$ & $10.05-15.09$ & $\mathbf{1 3}(1,2), \mathbf{1 5}(1,1), \mathbf{3 4}(1,1)$ \\
\hline 55. & Lycium barbarum $\mathrm{L}$. & $\mathrm{N}, \mathrm{P}$ & $20.05-20.08$ & $28(4,5)$ \\
\hline 56. & Malva neglecta Wallr. & $\mathrm{N}, \mathrm{P}$ & $15.06-15.09$ & $\mathbf{7}(3,5), \mathbf{9}(1,2), \mathbf{1 3}(1,1), \mathbf{1 5}(1,1), \mathbf{2 8}(1,1)$ \\
\hline 57. & $\begin{array}{l}\text { Matricaria maritima L. ssp. } \\
\text { inodora }\end{array}$ & $\mathrm{N}, \mathrm{P}$ & $10.05-20.09$ & $\mathbf{4}(1,1)$ \\
\hline 58. & Medicago falcata $\mathrm{L}$. & $\mathrm{N}, \mathrm{P}$ & $10.06-15.09$ & $\mathbf{1 2}(1,4), \mathbf{1 4}(1,1), \mathbf{1 5}(1,2), \mathbf{3 1}(1,3)$ \\
\hline 59. & Medicago lupulina $\mathrm{L}$. & $\mathrm{N}, \mathrm{P}$ & $10.06-15.08$ & $\begin{array}{l}\mathbf{6}(1,1), \mathbf{1 1}(1,1), \mathbf{1 2}(1,4), \mathbf{1 3}(1,1), \mathbf{1 4}(1,1), \mathbf{1 5}(1,2), \mathbf{3 1}(1,2), \\
\mathbf{3 2}(1,2), \mathbf{3 4}(1,2)\end{array}$ \\
\hline 60. & Medicago sativa $\mathrm{L}$. & $\mathrm{N}, \mathrm{P}$ & $10.06-15.08$ & $\mathbf{6}(2,1), \mathbf{1 3}(1,1), \mathbf{1 4}(1,1)$ \\
\hline 61. & Medicago xvaria Martyn & $\mathrm{N}, \mathrm{P}$ & $10.06-15.08$ & $7(2,1)$ \\
\hline 62. & $\begin{array}{l}\text { Melandrium album (Mill.) } \\
\text { Garcke }\end{array}$ & $\mathrm{N}, \mathrm{P}$ & $20.05-30.09$ & $\begin{array}{l}\mathbf{1}(1,2), \mathbf{3}(1,1), \mathbf{4}(1,1), \mathbf{7}(1,1), \mathbf{8}(1,2), \mathbf{1 2}(1,1), \mathbf{1 3}(1,3), \\
\mathbf{1 4}(1,2), \mathbf{1 5}(1,1), \mathbf{2 6}(1,2), \mathbf{2 8}(1,1)\end{array}$ \\
\hline 63. & Melilotus albus Medik. & $\mathrm{N}, \mathrm{P}$ & $10.06-15.08$ & $\mathbf{6}(1,1), \mathbf{1 2}(4,4), \mathbf{1 3}(1,1)$ \\
\hline 64. & Melilotus officinalis (L.) Pall. & $\mathrm{N}, \mathrm{P}$ & $01.06-20.07$ & $\mathbf{1 2}(1,1), \mathbf{1 4}(1,1)$ \\
\hline 65. & Myosotis arvensis (L.) Hill. & $\mathrm{N}, \mathrm{P}$ & $10.05-20.07$ & $2(1,1)$ \\
\hline 66. & Oenothera biennis L.s.s. & $\mathrm{N}, \mathrm{P}$ & $15.06-20.08$ & $\mathbf{1 3}(1,1), \mathbf{1 4}(1,1)$ \\
\hline 67. & Onopordum acathium $\mathrm{L}$. & $\mathrm{N}, \mathrm{P}$ & $15.06-20.07$ & $\mathbf{6}(1,1), \mathbf{1 1}(3,5)$ \\
\hline 68. & Papaver rhoeas $\mathrm{L}$. & $\mathrm{P}$ & $20.05-10.07$ & $\mathbf{1}(1,3), \mathbf{2}(2,2), \mathbf{3}(1,1), \mathbf{6}(1,2), \mathbf{1 4}(1,1), \mathbf{1 5}(1,1), \mathbf{2 0}(1,1)$ \\
\hline 69. & Pimpinella saxifraga $\mathrm{L}$. & $\mathrm{N}, \mathrm{P}$ & $15.06-30.09$ & $\begin{array}{l}\mathbf{6}(1,1), \mathbf{1 2}(1,3), \mathbf{1 3}(1,1), \mathbf{1 4}(1,1), \mathbf{1 5}(1,2), \mathbf{2 2}(1,1), \mathbf{3 2}(1,1), \\
\mathbf{3 4}(1,1)\end{array}$ \\
\hline 70. & Plantago lanceolata $\mathrm{L}$. & $\mathrm{P}$ & $15.05-10.09$ & $\mathbf{1 3}(1,3), \mathbf{1 4}(1,1), \mathbf{1 5}(1,2), \mathbf{3 2}(1,1), \mathbf{3 3}(1,1), \mathbf{3 4}(1,2)$ \\
\hline 71. & Plantago major L. & $\mathrm{P}$ & $20.05-15.08$ & $\begin{array}{l}\mathbf{3}(1,1), \mathbf{4}(1,3), \mathbf{7}(1,3), \mathbf{9}(2,2), \mathbf{1 3}(1,1), \mathbf{1 8}(1,1), \mathbf{3 2}(1,4), \\
\mathbf{3 3}(1,4), \mathbf{3 4}(2,4), \mathbf{3 5}(1,3)\end{array}$ \\
\hline 72. & Polygonum aviculare $\mathrm{L}$. & $\mathrm{N}, \mathrm{P}$ & $10.05-30.10$ & $\begin{array}{l}\mathbf{4}(1,5), \mathbf{5}(1,2), \mathbf{6}(1,1), \mathbf{7}(2,4), \mathbf{9}(2,3), \mathbf{1 0}(1,3), \mathbf{1 3}(1,1), \\
\mathbf{1 6}(1,1), \mathbf{3 5}(1,3)\end{array}$ \\
\hline
\end{tabular}




\begin{tabular}{|c|c|c|c|c|}
\hline 73. & Potentilla anserina $\mathrm{L}$. & $\mathrm{P}$ & $10.05-20.07$ & $\mathbf{4}(1,1), \mathbf{7}(1,1), \mathbf{3 2}(1,1), \mathbf{3 3}(3,5), \mathbf{3 4}(1,2)$ \\
\hline 74. & Prunella vulgaris L. & $\mathrm{N}, \mathrm{P}$ & $10.05-20.08$ & $\mathbf{3 4}(3,5)$ \\
\hline 75. & Ranunculus repens $\mathrm{L}$. & $\mathrm{P}$ & $10.06-10.08$ & $\mathbf{3 4}(1,1)$ \\
\hline 76. & Reseda lutea $\mathrm{L}$. & $\mathrm{N}, \mathrm{P}$ & $20.05-20.07$ & $\mathbf{1 5}(1,1)$ \\
\hline 77. & Reynoutria japonica Houtt. & $\mathrm{N}, \mathrm{P}$ & $05.08-30.09$ & 29(45) \\
\hline 78. & Robinia pseudoacacia L. & $\mathrm{N}, \mathrm{P}$ & $10.06-25.06$ & $\mathbf{2 4}(2,5), \mathbf{2 8}(1,2), \mathbf{3 0}(1,1)$ \\
\hline 79. & Rubus caesius L. & $\mathrm{N}, \mathrm{P}$ & $25.05-10.07$ & $\mathbf{1 3}(1,1), \mathbf{1 4}(1,2), \mathbf{1 5}(1,1), \mathbf{2 7}(1,2)$ \\
\hline 80. & Rumex acetosa $\mathrm{L}$. & $\mathrm{P}$ & $10.05-20.07$ & $\mathbf{1 3}(1,1), \mathbf{1 5}(1,1)$ \\
\hline 81. & Rumex crispus L. & $\mathrm{P}$ & $15.06-30.07$ & $\mathbf{6}(1,2), \mathbf{1 4}(1,1), \mathbf{1 5}(1,1), \mathbf{2 2}(1,1)$ \\
\hline 82. & Rumex obtusifolius L. & $\mathrm{P}$ & $15.06-15.07$ & $\mathbf{3}(1,1), \mathbf{1 1}(1,2), \mathbf{1 5}(1,1), \mathbf{1 6}(1,3), \mathbf{1 7}(1,3), \mathbf{2 2}(1,1), \mathbf{2 6}(1,2)$ \\
\hline 83. & Sambucus nigra L. & $\mathrm{P}$ & $20.05-20.06$ & $\begin{array}{l}\mathbf{1 4}(1,2), \mathbf{1 5}(1,1), \mathbf{1 6}(1,1), \mathbf{1 8}(1,2), \mathbf{2 0}(1,3), \mathbf{2 4}(1,3), \mathbf{2 6}(1,1), \\
\mathbf{2 8}(1,3), \mathbf{3 0}(1,5)\end{array}$ \\
\hline 84. & Saponaria officinalis & $\mathrm{N}, \mathrm{P}$ & $15.06-15.09$ & $\mathbf{2 4}(2,1), \mathbf{3 0}(1,1)$ \\
\hline 85. & Sedum acre $\mathrm{L}$. & $\mathrm{N}, \mathrm{P}$ & $15.05-20.07$ & $\mathbf{1 3}(1,1)$ \\
\hline 86. & Sisymbrium loeselii $\mathrm{L}$. & $\mathrm{N}, \mathrm{P}$ & $01.06-20.07$ & $\begin{array}{l}\mathbf{5}(1,2), \mathbf{6}(3,5), \mathbf{8}(1,3), \mathbf{9}(1,2), \mathbf{1 1}(2,1), \mathbf{1 4}(1,2), \mathbf{1 5}(1,2), \\
\mathbf{1 6}(1,2), \mathbf{1 9}(1,2), \mathbf{2 8}(1,1), \mathbf{3 0}(1,1), \mathbf{3 1}(1,2)\end{array}$ \\
\hline 87. & Solidago canadensis L. & $\mathrm{P}$ & $05.08-10.10$ & $\mathbf{6}(1,1), \mathbf{2 5}(2,1), \mathbf{2 6}(1,3), \mathbf{2 7}(1,1)$ \\
\hline 88. & Solidago gigantea Aiton & $\mathrm{N}, \mathrm{P}$ & $20.07-15.10$ & $\begin{array}{l}\mathbf{5}(1,5), \mathbf{1 0}(1,1), \mathbf{1 1}(2,1), \mathbf{1 2}(1,4), \mathbf{1 4}(4,5), \mathbf{1 5}(1,1), \mathbf{1 6}(1,2), \\
\mathbf{1 7}(1,2), \mathbf{1 9}(1,1), \mathbf{2 0}(2,2), \mathbf{2 2}(1,2), \mathbf{2 3}(1,1), \mathbf{2 9}(1,2), \mathbf{3 0}(1,1), \\
\mathbf{3 1}(1,2)\end{array}$ \\
\hline 89. & Sonchus arvensis L. & $\mathrm{N}, \mathrm{P}$ & $05.07-20.09$ & $\begin{array}{l}\mathbf{1}(1,1), \mathbf{2}(1,2), \mathbf{3}(1,2), \mathbf{6}(1,2), \mathbf{7}(1,4), \mathbf{9}(1,2), \mathbf{1 3}(1,2), \mathbf{1 4}(1,1), \\
\mathbf{1 5}(1,1), \mathbf{1 8}(1,2), \mathbf{1 9}(1,1), \mathbf{2 0}(1,1), \mathbf{2 1}(1,1), \mathbf{2 4}(1,2), \mathbf{2 6}(1,1), \\
\mathbf{2 9}(1,1), \mathbf{3 1}(1,2)\end{array}$ \\
\hline 90. & Symphytum officinale L. & $\mathrm{N}, \mathrm{P}$ & $15.05-20.08$ & $\mathbf{1 3}(1,1), \mathbf{2 6}(1,2)$ \\
\hline 91. & Tanacetum vulgare $\mathrm{L}$. & $\mathrm{P}$ & $20.07-01.10$ & $\begin{array}{l}\mathbf{5}(1,2), \mathbf{6}(1,1), \mathbf{8}(1,1), \mathbf{1 1}(2,1), \mathbf{1 2}(2,4), \mathbf{1 3}(1,2), \mathbf{1 4}(2,3), \\
\mathbf{1 5}(1,4), \mathbf{1 7}(1,2), \mathbf{2 1}(1,1), \mathbf{2 2}(1,2), \mathbf{2 9}(1,2), \mathbf{3 1}(1,3)\end{array}$ \\
\hline 92. & $\begin{array}{l}\text { Taraxacum officinale } \\
\text { F.H. Wigg. }\end{array}$ & $\mathrm{N}, \mathrm{P}$ & $05.05-25.05$ & $\begin{array}{l}\mathbf{1}(1,1), \mathbf{2}(1,1), \mathbf{3}(1,1), \mathbf{4}(1,4), \mathbf{5}(1,2), \mathbf{6}(2,1), \mathbf{7}(2,5), \mathbf{9}(1,4), \\
\mathbf{1 0}(1,3), \mathbf{1 2}(1,4), \mathbf{1 3}(1,3), \mathbf{1 4}(1,1), \mathbf{1 5}(1,3), \mathbf{1 6}(1,2), \mathbf{1 7}(1,1), \\
\mathbf{1 9}(1,1), \mathbf{2 1}(1,1), \mathbf{2 2}(1,2), \mathbf{2 3}(1,2), \mathbf{2 4}(1,4), \mathbf{2 8}(1,2), \mathbf{2 9}(1,1), \\
\mathbf{3 1}(1,5), \mathbf{3 2}(1,4), \mathbf{3 3}(2,2), \mathbf{3 4}(2,3), \mathbf{3 5}(1,2)\end{array}$ \\
\hline 93. & Trifolium pratense L. & $\mathrm{N}, \mathrm{P}$ & $01.06-30.07$ & $\begin{array}{l}\mathbf{1}(1,1), \mathbf{6}(1,1), \mathbf{7}(1,2), \mathbf{9}(1,2), \mathbf{1 4}(1,2), \mathbf{1 5}(1,3), \mathbf{1 9}(1,1), \\
\mathbf{3 1}(2,3), \mathbf{3 3}(1,1), \mathbf{3 4}(1,2)\end{array}$ \\
\hline 94. & Trifolium repens $\mathrm{L}$ & $\mathrm{N}, \mathrm{P}$ & $20.05-30.08$ & $\begin{array}{l}\mathbf{7}(2,3), \mathbf{1 2}(2,4), \mathbf{1 3}(1,1), \mathbf{1 5}(1,2), \mathbf{3 1}(2,3), \mathbf{3 2}(2,4), \mathbf{3 3}(1,1), \\
\mathbf{3 4}(1,4)\end{array}$ \\
\hline 95. & Tussilago farfara $\mathrm{L}$. & $\mathrm{N}, \mathrm{P}$ & $01.04-25.04$ & $\mathbf{1 9}(3,5)$ \\
\hline 96. & Verbascum nigrum $\mathrm{L}$. & $\mathrm{P}$ & $05.07-10.08$ & $\mathbf{1 4}(1,1), \mathbf{1 6}(1,1), \mathbf{1 7}(1,1)$ \\
\hline 97. & Vicia angustifolia $\mathrm{L}$ & $\mathrm{N}, \mathrm{P}$ & $10.05-15.08$ & $\mathbf{1 8}(1,1)$ \\
\hline 98. & Vicia cracca $\mathrm{L}$. & $\mathrm{N}, \mathrm{P}$ & $10.06-20.08$ & $\mathbf{1 3}(1,1), \mathbf{1 4}(1,2)$ \\
\hline 99. & Vicia sativa $\mathrm{L}$. & $\mathrm{N}, \mathrm{P}$ & $10.06-25.07$ & $\mathbf{2}(1,2), \mathbf{3}(1,1), \mathbf{1 5}(1,1)$ \\
\hline 100. & Vicia sepium L. & $\mathrm{N}, \mathrm{P}$ & $10.05-30.07$ & $\mathbf{1}(1,2)$ \\
\hline 101. & Vicia tetrasperma (L.) Schreb. & $\mathrm{N}, \mathrm{P}$ & $10.05-15.08$ & $\mathbf{1}(1,5), \mathbf{2}(1,1), \mathbf{6}(1,2), \mathbf{1 4}(1,1)$ \\
\hline 102. & Viola arvensis Murray & $\mathrm{N}, \mathrm{P}$ & $10.05-30.09$ & $\mathbf{1}(1,1), \mathbf{1 3}(1,1)$ \\
\hline
\end{tabular}

${ }^{1}$ Usefulness of plants to bumblebees: $\mathrm{N}$ - nectariferous species; $\mathrm{P}$ - polleniferous species.

${ }_{2}^{2}$ Plant communities: 1 - Vicietum tetrospermae, 2 - Echinochloo-Setarietum, $\mathbf{3}$ - Galinsogo-Setarietum, 4 - Panico-Eragrostietum, 5-Chenopodietum stricti, 6-Sisymbrietum loeselii, 7 - Urtico-Malvetum, 8-Erigeronto-Lactucetum, 9-Hordeetum murini, 10-Community with Lepidium ruderale, 11 - Onopordetum acanthii, 12 - Echio-Melilotetum, 13 - Berteroëtum incanae, 14 - ArtemisioTanacetetum, 15 - Bunietum orientalis, 16 - Leonuro-Ballotetum, $\mathbf{1 7}$ - Leonuro-Arctietum, $\mathbf{1 8}$ - Ivetum xantifolia, 19-Tussilaginetum, 20 - community with Cannabis ruderale, 21 - community with Heliantus tuberosus, 22 - Urtico-Aegopodietum, 23 - community with Impatiens parviflora, 24 - Chelidonio-Robinietum, 25 - Calystegio-Eupatorietum, 26 - Urtico-Calystegietum, 27 - community with Impatiens glandulifera, $\mathbf{2 8}$ - community with Lycium barbarum, 29 - zb. z Reynoutria japonica, $\mathbf{3 0}$ - Sambucetum nigrae, 31 - Cardario-Agropyretum, 32 - Lolio-Polygonetum, 33 - Lolio-Potentilletum, 34 - Prunello-Plantaginetum, 35 - Bryo-Saginetum. ${ }^{3}$ Cover-abundance: $1-1-20 ; 2-21-40 ; 3-41-60 ; 4-61-80 ; 5-81-100$.

${ }^{4}$ Sociability: 1 - single individuals; 2 - the species grows in groups or tufts; 3 - the species grows in tufts forming small patches; 4 - the species grows in extensive patches; 5 - pure population. 
Table 3

Flowering of bee plants in synanthropic plant communities found in Lublin

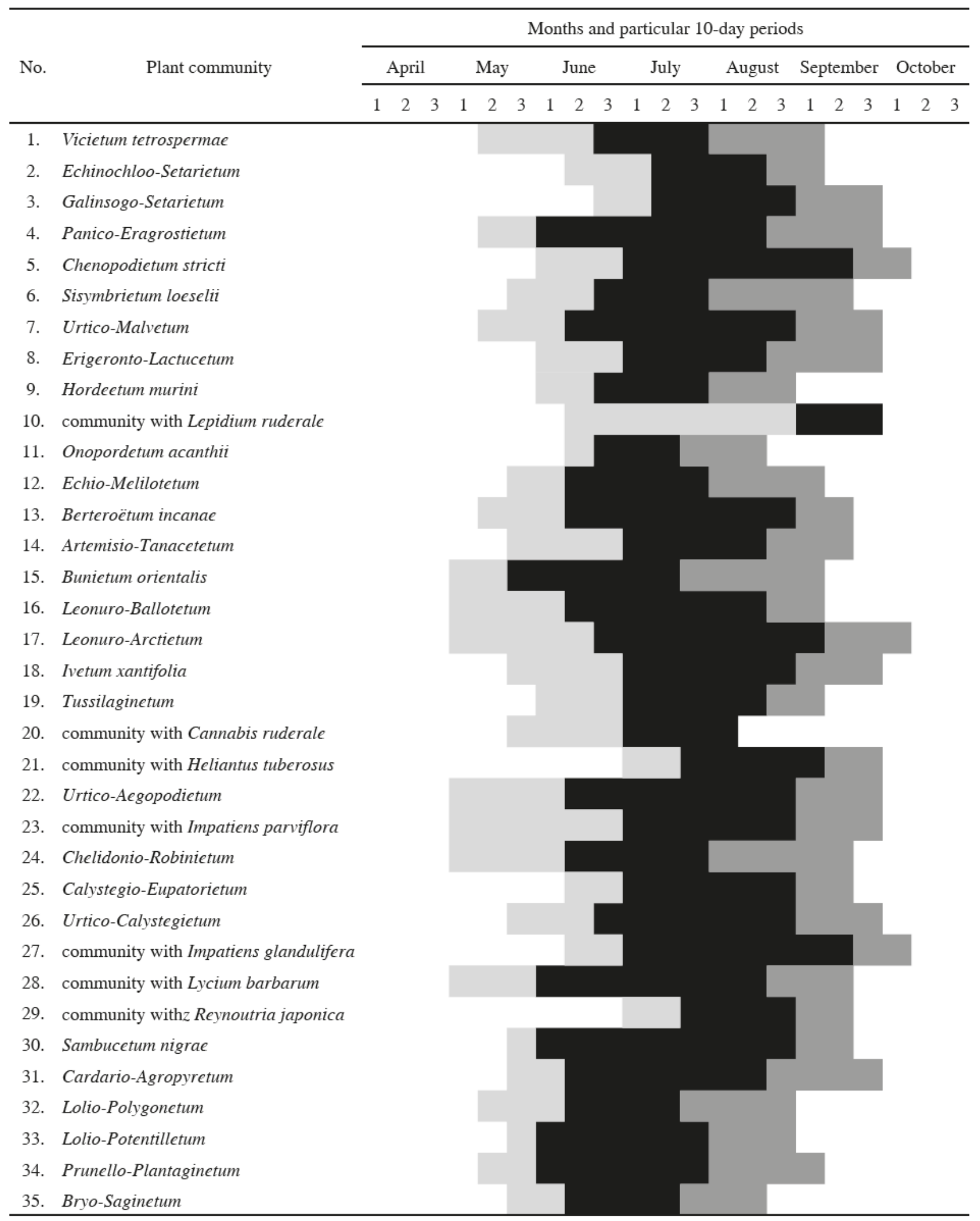

Early flowering (up to $39 \%$ bee plants bloom)

Full flowering (from 40 to $100 \%$ bee plants bloom)

Late flowering (up to $39 \%$ bee plants bloom) 
Table 4

Phenology of bumblebee species encountered in synanthropic communities in Lublin according to Dy lew ska [7]

\begin{tabular}{|c|c|c|c|c|c|c|c|c|c|c|c|c|c|c|c|c|c|c|c|c|c|}
\hline \multirow{3}{*}{$\begin{array}{l}\text { Bumblebee } \\
\text { species }\end{array}$} & \multirow{3}{*}{ Caste } & \multicolumn{20}{|c|}{ Months and particular 10-day periods } \\
\hline & & \multicolumn{2}{|c|}{ March } & \multicolumn{3}{|c|}{ April } & \multicolumn{2}{|c|}{ May } & \multicolumn{3}{|c|}{ June } & \multicolumn{3}{|c|}{ July } & \multicolumn{3}{|c|}{ August } & \multicolumn{2}{|c|}{ September } & \multicolumn{2}{|c|}{ October } \\
\hline & & 12 & 3 & 1 & 2 & 3 & 1 & 2 & 31 & 2 & 3 & 1 & 2 & 3 & 1 & 2 & 3 & 12 & 3 & 12 & 3 \\
\hline \multirow{4}{*}{$\begin{array}{l}\text { Buff-tailed } \\
\text { bumblebee } \\
\text { B. terrestris }\end{array}$} & queens & & & & & & & & & & & & & & & & & & & & \\
\hline & workers & & & & & & & & & & & & & & & & & & & & \\
\hline & males & & & & & & & & & & & & & & & & & & & & \\
\hline & young queens & & & & & & & & & & & & & & & & & & & & \\
\hline \multirow{4}{*}{$\begin{array}{l}\text { Red-tailed } \\
\text { bumblebee } \\
\text { B. lapidarius }\end{array}$} & queens & & & & & & & & & & & & & & & & & & & & \\
\hline & workers & & & & & & & & & & & & & & & & & & & & \\
\hline & males & & & & & & & & & & & & & & & & & & & & \\
\hline & young queens & & & & & & & & & & & & & & & & & & & & \\
\hline
\end{tabular}

\section{DISCUSSION}

Synanthropic vegetation can be an important element of food resources for bumblebees. These insects readily use herbaceous plants as a source of food [53]. The present study found Artemisio-Tanacetetum, Bunietum orientalis and Berteroëtum incanae to be the most important synanthropic communities to bumblebees in Lublin, since the largest number of nectariferous and polleniferous species was recorded in them. Moreover, in Lublin these communities occur in different parts of the city and occupy large areas. As reported by Banaszak [29] as well as by Wrzesień and D e $n$ is ow [26], the maintenance of a mosaic of phytocenoses in anthropogenically transformed habitats is of great importance in the conservation of biological diversity, including the protection of habitats of Apidae. Among plants that were found in synanthropic communities of Lublin, Te per $[54,55]$ showed the following taxa as food plants important to B. terrestris: Cichorium sp., Centaurea cyanus, Echium vulgare, Helianthus sp., Heracleum sp., Lotus corniculatus, Melilotus sp., Plantago sp., Taraxacum sp., Trifolium pratense, Trifolium repens, Rubus sp., Solidago sp., Symphytum sp., and Vicia sp. Apart from the abovementioned plants, Tilia sp., Lythrum sp. and plants of the family Brassicacae proved to be important to this bumblebee species. Therefore, the results of $\mathrm{Te}$ per $[54,55]$ confirm the thesis about the major importance of synanthropic vegetation as a source of food for bumblebees.

This study confirms that urban environment, including synanthropic vegetation, is an important factor for bumblebees, determining their survival. In Lublin the study showed the clear presence of $B$. terrestris and $B$. lapidarius as well as of $B$. lucorum, B. hypnorum and B. pascuorum. Other authors have reported similar data for other cities of Poland [10,16,30,32,56]. In Warsaw B a n a $\mathrm{z}$ a k [10] showed the presence in large numbers of B. terrestris, B. lucorum, and B. hypnorum. Kowalczyk et al. [16] showed the occurrence of the following species in the city centre of Łódź: Bombus hortorum (Linnaeus 1761), B. hypnorum, B. lapidarius, B. lucorum, B. pascuorum, Bombus pratorum (Linnaeus 1761), Bombus ruderarius (Muller 1776), B. terrestris, and Bombus norvegicus (Sparre-Schneider 1918). On the other hand, in Kielce the following bumblebee species occurred in greatest numbers: $B$. hypnorum, B. lapidarius, B. lucorum, B. pascuorum, B. ruderarius, and B. terrestris [56]. In Poznań the following were found: $B$. hypnorum, B. pascuorum, and $B$. terrestris [30], whereas in Torun $B$. terrestris, $B$. pascuorum, B. lapidarius, B. hypnorum and B. lucorum were the most numerous [32]. Bombus terrestris and Bombus lapidarius occur in greatest numbers in urban areas both in Lublin and in other regions of Poland $[5,7,15-17,32]$.

The importance of synanthropic plant communities found in Lublin to bumblebees results from their high species diversity as well as from the presence of polleniferous and nectariferous species in large numbers. Furthermore, the area occupied by these communities, their location and association with other green spaces play an important role. For this reason, it is so important to maintain the existing communities and to provide extensive lawn maintenance. And even, as claimed by $\mathrm{M} \mathrm{e} \mathrm{z}$ et al. [57], bridge plants, i.e. plants planted and maintained to attract pollinating insects and to maintain their population, should be introduced in degraded ecosystems for pollinators with special trophic requirements. 
As mentioned above, it is presumed that polylectism of bees, i.e. visiting flowers of unrelated plants, is also an important feature that facilitates their survival in the anthropogenic environment [30]. B a $\mathrm{n}$ as zak-Cibicka [30] includes Apis melifera L. and members of the genus Bombus in bees characterized by polylectism. Thus, given that bumblebees can collect food from many unrelated plants, a conclusion then arises that such species-diverse synanthropic communities of Lublin have a major role for bumblebees.

Bumblebees are very efficient pollinators and besides they are less sensitive to changes in atmospheric conditions than A. melifera, especially to a decrease in temperature and an increase in air humidity. It is presumed that the work of one bumblebee is equal to the work of 4-5 honey bee workers [5]. The role of bumblebees as pollinators in human economy and in the maintenance of the diversity of entomophilous flora is invaluable and therefore it seems so important to maintain the largest possible number of environments that they can inhabit. The phenomenon of mass die-off of A. melifera, termed the CCD (Colony Collapse Disorder) syndrome, has been observed in the world since 2006. Over a period of 4 years, the numbers of bees in Germany and France decreased by $40 \%$, while in some regions of the USA even by $90 \%$. In Poland this phenomenon is not observed with such intensity, but the first symptoms can already be noticed [58]. Therefore, apart from the research designed to find the reasons for the CCD syndrome and to prevent it, simultaneously it is important to protect pollinators, including bumblebees.

It is worth maintaining habitats that will serve bumblebees in their development. The importance of urban areas as unique fauna reservoirs is continually increasing [14,17]. Under the Convention on Biological Diversity, biodiversity conservation is included in the state's tasks and hence it is also important to protect ecosystems and the continuity of biological process in cities. Cities do not have vast habitat areas and in cities there are few such biologically diverse places as synanthropic communities. In wasteland, animals can find breeding sites and valuable trophic areas, since well-tended green spaces will not play such a role. Most bumblebee species found in Poland build their nests in the soil [5,7]. It is not possible for them to locate their nests in intensely mowed lawns or carefully tended flower borders. Therefore, synanthropic communities have a major role to play again; as natural open areas, they can be perfect habitats for bumblebees.

The maintenance of spontaneous synanthropic vegetation in urban areas enables the conservation of biodiversity at different levels and leaving these habitats creates conditions for living and development of many animal species, including useful and protected species such as bumblebees.

\section{Acknowledgments}

Research supported by the activities of the Institute of Landscape Architecture, John Paul II Catholic University of Lublin.

\section{Authors' contributions}

The following declarations about authors' contributions to the research have been made: concept of the study ML, ET, field work: ML, ET, writing: ML, ET.

\section{REFERENCES}

1. Fauna Europaea version 2.5. [Internet]. 2012 [Cited 2012 June 11]; Available from: http://www.faunaeur.org

2. B a naszak J. Apidae. [In:] Bogdanowicz W, Chudzicka E, Pilipiuk I, Skibińska E, editors Fauna Polski. Charakterystyka i wykaz gatunków. Fauna of Poland. Characteristic and checklist of species. Warszawa: MiIZ PAN. 2004: 358-362. (in Polish)

3. Rozporządzenie Ministra Środowiska z dnia 12 października 2011 roku w sprawie ochrony gatunkowej zwierząt [Internet]. 2011 [Cited 2012 June 11]; Available from: http://isap.sejm.gov.pl/DetailsServlet?id=WDU2011237 1419 (in Polish)

4. B a na s zak J. O ochronę gatunkową wszystkich pszczół dziko żyjących (Hymenoptera, Apoidea). / For the conservation of all wild bee species (Hymenoptera, Apoidea). Chrońmy przyrodę ojczystą. 1990; 1: 5-8. (in Polish)

5. Banaszak J. Trzmiele Polski. Bydgoszcz: Wyższa Szkoła Pedagogiczna w Bydgoszczy. 1993. (in Polish)

6. Kosior A. Changes in the fauna of bumble-bees (Bombus Latr.) and cuckoo-bees (Psithyrus Lep.) of selected regions in Southern Poland. [In:] Banaszak J. editor. Changes in Fauna of Wild Bees in Europe. Pedagogical University, Bydgoszcz. 1995: 103-114.

7. D y lew ska M. Nasze trzmiele. Karniowice: Ośrodek Doradztwa Rolniczego; 1996. (in Polish)

8. Sárospataki M, Novák J, Molnár W. Assessing the threatened status of bumble bee species (Hymenoptera, Apidae) in Hungary, Central Europe. Biodiversity and Conservation. 2005; 14: 2437-2446. http://dx.doi.org/10.1007/ s10531-004-0152-y

9. Gouls on D. Bumblebees; their behaviour, ecology and conservation. Oxford: Oxford University Press. 2010.

10. B a n a s z a k J. Bees of urban environments in Poland. [In:] Banaszak J. (ed.) Natural Resources of Wild Bees in Poland. Bydgoszcz: Pedagogical University. 1992: 101-107.

11. Kle m m M. Man-made bee habitats in the anthropogenous landscape of central Europe - substitutes for threatened or destroyed riverine habitats? [In:] Matheson A, Buchmann SL, O'Toole C, Westrich P, Williams IH, (eds) The Conservation of Bees. London: Academic Press. 1996: 17-34.

12. Goulson D, Hughes WOH, Derwent LC, Stout JC. Colony growth of the bumble bee, Bombus terrestris, in improved and conventional agricultural and 
suburban habitats. Oecologia. 2002. 130: 267-273. http://dx. doi.org/10.1007/s004420100803

13. Goulson D. Bumble bees - behaviour and ecology. Oxford: Oxford University Press. 2003.

14. McFrederick QS, LeBuhn G. Are urban parks refuges for bumblebees Bombus spp. (Hymenoptera: Apidae)?. Biological Conservation. 2006; 129(3): 372-382. http://dx.doi.org/10.1016/j.biocon.2005.11.004

15. B a naszak J. Fauna pszczół (Hymenoptera: Apoidea: Apiformes) Bydgoszczy. I The bee fauna (Hymenoptera: Apoidea: Apiformes) of Bydgoszcz. [In:] Indykiewicz P, Jerzak L, Barczak T, editors. Fauna miast. Ochronić różnorodność biotyczną w miastach. Bydgoszcz: SAR "Pomorze". 2008; 234-245. (in Polish)

16. Kowalczyk JK, Szczepko K, Kurzac T. Stan poznania pszczół (Hymenoptera, Apoidea, Apiformes) Łodzi. [In:] Indykiewicz P, Jerzak L, Barczak T, editors. Fauna miast. Ochronić różnorodność biotyczną w miastach. Bydgoszcz: SAR "Pomorze". 2008; 246-252. (in Polish)

17. Banaszak-Cibicka W, B anaszak J. Pollinating insects of cities (Hymenoptera: Apoidea: Apiformes). Part I. Fauna of Poznan in comparison with other polish cities. In: Indykiewicz P, Jerzak L, Böhner J, Kavanagh B, editors. Urban fauna. Studies of animal biology, ecology and conservation in European cities. Bydgoszcz: UTP. 2011; 227-236.

18. Lye GC, Osborne JL, Park KJ, Goulson D. Using citizen science to monitor Bombus populations in the UK: nesting ecology and relative abundance in the urban environment. J Insect Conserv. 2012; 16(5): 697-707. http://dx.doi.org/10.1007/s10841-011-9450-3

19. McIntyre NE, Hostetler ME. Effects of urban land use on pollinator (Hymenoptera: Apoidea) communities in a desert metropolis. Basic and Applied Ecology. 2001; 2(3): 209-218. http://dx.doi.org/10.1078/1439-1791-00051

20. Hennig EI, Ghazoul J. Pollinating animals in the urban environment. Urban Ecosyst. 2012; 15: 149-166. http://dx.doi.org/10.1007/s11252-011-0202-7

21. Hopwood JL. The contribution of roadside grassland restorations to native bee conservation. Biological Conservation. 2008; 141(10): 2632-2640. http://dx.doi.org/10.1016/j.biocon.2008.07.026

22. Buszko J, Masłowski J. Atlas motyli Polski. Część I Motyle dzienne (Rhopalocera). Warszawa: Grupa IMAGE. 1993. (in Polish)

23. B u s z k o J . Atlas motyli Polski Część II Prządki, zawisaki, niedźwiedziówki, Warszawa: Grupa IMAGE. 1997. (in Polish)

24. B u s z k o J . Atlas motyli Polski Część III Falice, wycinki, miernikowce, Warszawa: Grupa IMAGE. 2000. (in Polish)

25. Eremeeva N I, Sushchev DV. Structural Changes in the Fauna of Pollinating Insects in Urban Landscapes. Russian Journal of Ecology. 2005; 36(4) 259-265. http://dx.doi. org/10.1007/s11184-005-0070-6

26. Wrzesień M, Den is ow B. The phytocoenoses of anthropogenically transformed areas with a great importance for Apoidea. Acta Agrobot. 2007; 60(2): 117-126.
27. Öckinger E, Dannestam A, Smith HG. The importance of fragmentation and habitat quality of urban grasslands for butterfly diversity. Landscape and Urban Planning. 2009; 93: 31-37. http://dx.doi.org/10.1016/j. landurbplan.2009.05.021

28. Bergerot B, Fontaine B, Renard M, Cadi A, Julliard R. Preferences for exotic flowers do not promote urban life in butterflies. Landscape and Urban Planning. 2010; 96(2): 98-107. http://dx.doi.org/10.1016/j. landurbplan.2010.02.007.

29. B a n a szak J. Pszczoły środowisk miejskich. [In:] Barczak T, Indykiewicz P, editors. Fauna miast - Urban fauna. Bydgoszcz: Wyd. ATR. 1998; 57-62. (in Polish)

30. B a naszak-Cibicka W. Wstępne informacje o zróżnicowaniu fauny pszczół Poznania (Hymenoptera: Apoidea: Apiformes). [In:] Indykiewicz P, Jerzak L, Barczak T, editors. Fauna miast. Ochronić różnorodność biotyczną w miastach. Bydgoszcz: SAR "Pomorze. 2008; 260-264. (in Polish)

31. Ahrné K, Bengtsson J, Elmqvist T. Bumble Bees (Bombus spp) along a Gradient of Increasing Urbanization. PLoS. 2009; 4(5): e5574: 1-9. http://dx.doi.org/10. 1371/journal.pone.0005574

32. Pawlikowski T, Olędzka J. Atrakcyjność środowisk miejskich dla trzmieli (Hymenoptera, Apidae) na obszarze Torunia. Wiad Entomol. 1996; 15(2): 97-103. (in Polish)

33. Araujo ED, Costa M, Chaud-Netto J, Fowler HG. Body size and flight distance in stingless bees (Hymenoptera: Meliponini): Inference of flight range and possible ecological implications. Braz J Biol. 2004; 64(3B): 563-568. http://dx.doi.org/10.1590/S1519-69842004000400003

34. Greenleaf SS, Williams NM, Winfree R, $\mathrm{Kremen} C$. Bee foraging ranges and their relationship to body size. Oecologia. 2007; 153: 589-596. http://dx.doi. org/10.1007/s00442-007-0752-9

35. Gathmann A, Tscharntke T. Foraging ranges of solitary bees. J Anim Ecol. 2002; 71: 757-764. http://dx.doi. org/10.1046/j.1365-2656.2002.00641.x

36. Steffan-Dewenter I, Munzenberg U, Burger C, Thies C., Tscharntke T. Scale-dependent effects of landscape context on three pollinator guilds. Ecology. 2002; 83: 1421-1432. http://dx.doi.org/10.1890/0012-9658 (2002)083[1421:SDEOLC]2.0.CO;2

37. Steffan-Dewenter I, Kuhn A. Honeybee foraging in differentially structured landscapes. Proc. Biol. Sci. 2003; 270: 569-575. http://dx.doi.org/10.1098/rspb.2002. 2292

38. Wojcik VA, McBride JR. Common factors influence bee foraging in urban and wildland landscapes. Urban Ecosyst. 2012; 15: 581-598. http://dx.doi.org/10.1007/ s11252-011-0211-6

39. Wolf S, Moritz RFA. Foraging distance in Bombus terrestris L. (Hymenoptera: Apidae) Apidologie. 2008; 39: 419-427. http://dx.doi.org/10.1051/apido:2008020

40. Masierowska M. Some ornamental shrubs as food source for pollinating insects. [In:] Banaszak J, editor. Changes 
in Fauna of Wild Bees in Europe. Bydgoszcz: Pedagogical University. 1995; 189-200.

41. Wrzesién M, Denis ow B. The share of nectariferous and polleniferous taxons in chosen patches of thermophilous grasslands of the Lublin Upland. Acta Agrobot. 2006; 59(1): 213-221.

42. Wrzesień M, Denisow B. The usable taxons in spontaneous flora of railway areas of the central-eastern part of Poland. Acta Agrobot. 2006; 59(2): 95-108.

43. Denis ow B, Wrzesień M. The anthropogenic refuge areas for bee flora in agricultural landscape. Acta Agrobot. 2007; 60(1): 147-157.

44. Pawlikowski T. Przewodnik terenowy do oznaczania trzmieli i trzmielców Polski (Hymenoptera: Apidae: Bombini). Toruń: Wyd. UMK. 1999. (in Polish)

45. Li pi ń sk i M. Pożytki pszczele, zapylanie i miododajność roślin. Warszawa: PWRiL. 2010. (in Polish)

46. Matus zk i ew i c W. Przewodnik do oznaczania zbiorowisk roślinnych Polski. Warszawa: PWN. 2004. (in Polish)

47. Fijałkowski D. Zbiorowiska roślin synantropijnych miasta Lublina. Ann UMCS Sec. C. 1967; 22 (17): 195230. (in Polish)

48. J a n e cki J. Człowiek a roślinność synantropijna na przykładzie Warszawy. Warszawa: Wyd. SGGW-AR. 1983. (in Polish)

49. Alford DV. Bumblebees. London: Davis-Poynter; 1975.

50. Heinrich B. Bumblebee Economics with a new preface (Revised edition). Cambridge: Harvard University Press. 2004.

51. Rysiak A. Extinct species in the vascular plant flora of Lublin city (E Poland). [In:] Mirek Z, Nikiel A, (eds) Rare, relict and endangered plants and fungi in Poland. Kraków: Wladyslaw Szafer Institute of Botany of the Polish Academy of Sciences. 2009; 443-455.

52. Trzaskowska E. Wykorzystanie roślin i zbiorowisk synantropijnych na terenach zieleni Lublina. Lublin: Wyd KUL. 2013; (in Polish)

53. Kelm M, Biesiada A, Krawczyk M, Ciołek M. Atrakcyjność kwiatów wybranych roślin zielarskich dla trzmieli (Apoidea: Bombus spp.). Zesz Probl Postępów Nauk Rol. 2011; 562: 71-76. (in Polish)
54. Teper D. Food plants of Bombus terrestris as determined by polinological analysis of pollen loads. J Apicul Sci. 2004; 48(2): 75-81.

55. Teper D. Food plants of Bombus terrestris as determined by pollen analysis of faeces. J Apicul Sci. 2006; 50(2): 101-108.

56. B ą k J. Tereny zieleni miejskiej Kielc - miejscem atrakcyjnym dla trzmieli (Hymenoptera, Apiformes, Bombini). [In:] Indykiewicz P, Jerzak L, Barczak T, (eds) Fauna miast. Ochronić różnorodność biotyczną w miastach. Bydgoszcz: SAR "Pomorze". 2008; 265-272. (in Polish)

57. Menz MHM, Phillips RD, Winfree R, Kremen C, Aizen MA, Johnson SD, et al. Reconnecting plants and pollinators: challenges in the restoration of pollination mutualisms. Trends Plant Sci. 2011; 16(1): 4-12. http://dx.doi.org/10.1016/j.tplants.2010.09.006.

58. Kupczyk M. Zginą pszczoły zginie ludzkość. [In:] Dziek. J, Chmiel J, (eds) To nie przyroda jest na krawędzi. Barcin: Stowarzyszenie Ekologiczne. 2009; 41-49. (in Polish)

\section{Potencjalne znaczenie roślinności synantropijnej dla trzmieli w ekosystemach miejskich na przykładzie Lublina}

\section{Streszczenie}

W pracy podjęto próbę przedstawienia potencjalnie cennych fitocenoz synantropijnych Lublina dla owadów zapylających jakimi są trzmiele. Na terenie Lublina stwierdzono występowanie $B$. terrestris i B. lapidarium, a także B. lucorum, B. hypnorum i B. pascuorum. Ze względu na stwierdzoną wysoką liczbę gatunków pyłkodajnych i nektarodajnych do najcenniejszych dla trzmieli zaliczono: Artemisio-Tanacetetum, Bunietum orientalis, Berteroëtum incanae. Przeprowadzone badania pozwoliły również określić, że na rozmieszczenie trzmieli ma wpływ wielkość i występowanie zbiorowisk $\mathrm{w}$ mozaice $\mathrm{z}$ parkami i terenami zieleni.

Handling Editor: Elżbieta Pogroszewska

This is an Open Access digital version of the article distributed under the terms of the Creative Commons Attribution 3.0 License (creativecommons.org/licenses/by/3.0/), which permits redistribution, commercial and non-commercial, provided that the article is properly cited.

CThe Author(s) 2013 Published by Polish Botanical Society 